УДК 512.541

\title{
On Determinability of a Quotient Divisible Abelian Group of Rank 1 by Its Automorphism Group
}

\author{
Vadim K. Vildanov* \\ Lobachevsky State University of Nizhni Novgorod \\ Gagarina, 23, Nizhni Novgorod, 603950 \\ Russia \\ Egor A. Timoshenko ${ }^{\dagger}$ \\ Tomsk State University \\ Lenina, 36, Tomsk, 634050 \\ Russia
}

Received 13.02.2019, received in revised form 22.07.2019, accepted 20.09.2019

A criterion for determinability of a quotient divisible Abelian group of rank 1 by its automorphism group in the class of quotient divisible Abelian groups of rank 1 is considered in the paper.

Keywords: determinability, quotient divisible group, automorphism group.

DOI: 10.17516/1997-1397-2019-12-6-699-704.

The word "group" will mean an Abelian group. As usual, $A$ is a torsion group (a torsion-free group) if $\mathrm{t}(A)=A(\mathrm{t}(A)=0)$, where $\mathrm{t}(A)$ is the subgroup of all elements of finite order in $A$. A nonzero group $A$ is a torsion-free group of rank 1 if it is isomorphic to a subgroup of $\mathbb{Q}$; we say that this group is a rank 1 torsion-free group of idempotent type if $A$ is isomorphic to the additive group of some subring of $\mathbb{Q}$.

Let $A \in \mathbf{X}$, where $\mathbf{X}$ is some class of groups. We say that $A$ is determined by its automorphism group in the class $\mathbf{X}$ if Aut $A \cong$ Aut $B$ implies $A \cong B$ for every $B \in \mathbf{X}$.

It was proved that a $p$-group is determined by its automorphism group in the class of all $p$-groups for $p \geqslant 5$ [1]. Similar result was obtained for $p=3$ [2]. Rank 1 torsion-free groups are not determined by their automorphism groups, but for direct sums of torsion-free groups of rank 1 (i.e., for completely decomposable torsion-free groups) necessary and sufficient conditions for determinability of a group by its automorphism group in some classes were found (see [3, 4]). Among other things, the question of determinability of a group by its automorphism group was considered in the class of completely decomposable torsion-free groups whose direct summands of rank 1 have idempotent types [3]. In particular it was shown that a torsion-free group $A$ of rank 1 and of idempotent type is determined by its automorphism group in the class of all such groups if and only if $A$ is an infinite cyclic group. Let us note that the class of rank 1 torsion-free groups of idempotent type coincides with the class of quotient divisible groups of rank 1 which are torsion-free.

Let us denote the set of all primes by $\mathbb{P}$. Recall (for example, see [5]) that a group $A$ is $p$-divisible if the set $p A=\{p a \mid a \in A\}$ coincides with $A$; a divisible group is a group which is $p$-divisible for all $p \in \mathbb{P}$.

\footnotetext{
*kadirovi4@gmail.com

†tea471@mail.tsu.ru

(c) Siberian Federal University. All rights reserved
} 
Definition. Suppose that a group $A$ does not contain nonzero divisible torsion subgroups and there is an element $x \in A$ of infinite order such that $A /\langle x\rangle$ is a divisible torsion group. Then $A$ is called the quotient divisible group of rank 1 and $x$ is called the basis element of this group.

For an arbitrary quotient divisible group $A$ of rank 1 we define a characteristic $\chi=\left(m_{p}\right)_{p \in \mathbb{P}}$ by taking $m_{p}$ to be the smallest number $m \geqslant 0$ for which $p^{m} x$ is in $p^{n} A$ for all $n>0$ (if there are no such numbers, we set $m_{p}=\infty$ ). We say that $\chi$ is the cocharacteristic of the element $x$. The cocharacteristic of a basis element of the group $A$ is uniquely determined by $A[6]$ and it is called the cocharacteristic of the group A.

The class of all quotient divisible groups of rank 1 is denoted by $\mathcal{Q D} \mathcal{D}_{1}$. Let us recall the structure of quotient divisible groups of rank 1 (see $[6,7])$. Let $\chi=\left(m_{p}\right)_{p \in \mathbb{P}}$ be an arbitrary characteristic and $L$ be the set of all $p \in \mathbb{P}$ for which $0<m_{p}<\infty$. We denote the direct product of all rings $\mathbb{Z} / p^{m_{p}} \mathbb{Z}$ with $p \in L$ by $K^{\chi}$ and we also denote the subring of the field $\mathbb{Q}$ generated by the elements $\frac{1}{p}$ such that $m_{p}<\infty$ by $\mathbb{Q}^{\chi}$ (if all $m_{p}$ are infinite then $\mathbb{Q}^{\chi}=\mathbb{Z}$ ). If $L$ is a finite set, we define $R^{\chi}=\mathbb{Q}^{\chi} \times K^{\chi}$.

If the set $L$ is infinite then $R^{\chi}$ is the ring of all elements $b=\left(b_{p}\right)_{p \in L} \in K^{\chi}$ such that for some fraction $\frac{u}{v} \in \mathbb{Q}^{\chi}$ the equality $u e_{p}=v b_{p}$ holds for almost all $p \in L$, where $e_{p}$ is the identity of the ring $\mathbb{Z} / p^{m_{p}} \mathbb{Z}$ ). The mapping $\pi$ that assigns to every such element $b$ the number $\frac{u}{v}$ is an epimorphism of $R^{\chi}$ onto $\mathbb{Q}^{\chi}$. Its kernel Ker $\pi$ coincides with the direct $\operatorname{sum} \mathrm{t}\left(R^{\chi}\right)$ of all $\mathbb{Z} / p^{m_{p}} \mathbb{Z}$ with $p \in L$.

It can be checked directly that for any characteristic $\chi$ the additive group of the ring $R^{\chi}$ is a quotient divisible group of rank 1, while the identity of the ring is the basis element of this group and it has $\chi$ as its cocharacteristic. It follows that any quotient divisible group of rank 1 with cocharacteristic $\chi$ is isomorphic to the additive group of the ring $R^{\chi}$ [6]. In particular, if $A \in \mathcal{Q D}_{1}$ then the factor group of $A$ with respect to its torsion part $\mathrm{t}(A)$ is a rank 1 group of idempotent type which is $p$-divisible for every $p \in \mathbb{P}$ such that the $p$-component $A_{p}$ is nonzero.

The symbol $\prod$ denotes a restricted direct product of groups. This analogue of the notion of a direct sum is used for multiplicatively written groups.

Proposition 1. If $A$ is a quotient divisible group of rank 1 , then

$$
\text { Aut } A \cong \operatorname{Aut}(A / \mathrm{t}(A)) \times \prod_{p \in L} \operatorname{Aut} A_{p}
$$

where $L$ is the set of all $p \in \mathbb{P}$ for which $A_{p} \neq 0$.

Proof. We may assume that $A$ coincides with one of the rings $R^{\chi}$. If $L$ is finite then $A$ is the direct sum of the $p$-components $A_{p}$ with $p \in L$ and of the group $\mathbb{Q}^{X} \cong A / \mathrm{t}(A)$ which is $p$-divisible for every $p \in L$. Since each of these direct summands is fully invariant in $A$, we arrive at the isomorphism $(*)$.

Now let the set $L$ be infinite. It is known that any endomorphism of the additive group of the ring $A=R^{\chi}$ is a multiplication by some element of $A[6,7]$. From this we obtain Aut $A \cong A^{*}$, where $A^{*}$ is the group of invertible elements of the ring $A$. It is easy to verify that the restriction $\rho$ of the epimorphism $\pi: A \rightarrow \mathbb{Q}^{x}$ to the group $A^{*}$ has the group $\left(\mathbb{Q}^{\chi}\right)^{*}$ as its image. Its kernel Ker $\rho$ is the set of elements $b=\left(b_{p}\right)_{p \in L} \in A^{*}$ such that $b_{p}=e_{p}$ for almost all $p \in L$.

Let us show that Ker $\rho$ is a pure subgroup of $A^{*}$. Suppose that $b=\left(b_{p}\right)_{p \in L}$ is an element of the group $A^{*}$ such that $b^{n} \in \operatorname{Ker} \rho$ (for some natural $n$ ). Let us define the element $c=\left(c_{p}\right)_{p \in L} \in A^{*}$ 
by setting $c_{p}=b_{p}$ if $\left(b_{p}\right)^{n} \neq e_{p}$ and $c_{p}=e_{p}$ otherwise. Obviously $c^{n}=b^{n}$ and $c \in \operatorname{Ker} \rho$ as desired.

$\operatorname{Im} \rho=\left(\mathbb{Q}^{\chi}\right)^{*}$ is a restricted direct product of cyclic groups. Using Theorem 28.2 [5], we have $A^{*} \cong\left(\mathbb{Q}^{\chi}\right)^{*} \times \operatorname{Ker} \rho$. Then

$$
\text { Aut } A \cong\left(\mathbb{Q}^{\chi}\right)^{*} \times \operatorname{Ker} \rho \cong \operatorname{Aut} \mathbb{Q}^{\chi} \times \prod_{p \in L}\left(A_{p}\right)^{*} \cong \operatorname{Aut}(A / \mathrm{t}(A)) \times \prod_{p \in L} \operatorname{Aut} A_{p}
$$

This completes the proof of the proposition.

Cyclic groups of infinite order and of order $n$ are denoted by $Z$ and $Z(n)$, respectively. It makes no difference whether these groups are written additively or multiplicatively.

Proposition 2. Suppose all p-components of a quotient divisible group A of rank 1 are nonzero. Then

$$
\text { Aut } A \cong \prod_{\aleph_{0}} Z \times \prod_{q \in \mathbb{P}} \prod_{k>0} \prod_{\aleph_{0}} Z\left(q^{k}\right)
$$

Proof. It follows from the description of quotient divisible groups of rank 1 that $A / \mathrm{t}(A) \cong \mathbb{Q}$. Using Proposition 1 and decomposing each group Aut $A_{p}$ into the product of indecomposable cyclic subgroups, we obtain that

$$
\text { Aut } A \cong \prod_{\aleph_{0}} Z \times \prod_{q \in \mathbb{P}} \prod_{k>0} \prod_{\mathfrak{J}_{q, k}} Z\left(q^{k}\right),
$$

where $\mathfrak{J}_{q, k} \leqslant \aleph_{0}$ for every $q \in \mathbb{P}$ and $k>0$.

We fix $q^{k}$ and consider the arithmetic progression with initial term $1+q^{k}$ and difference $q^{k+1}$. By the Dirichlet theorem (see [8]) this progression contains infinitely many primes. If $p$ is one of such primes and $A_{p} \cong Z\left(p^{m}\right)$ then Aut $A_{p} \cong Z(p-1) \times Z\left(p^{m-1}\right)$ has a direct factor $Z\left(q^{k}\right)$ since $p-1$ is divisible by $q^{k}$ but not by $q^{k+1}$. It follows that each of the cardinals $\mathfrak{J}_{q, k}$ is equal to $\aleph_{0}$. This proves the proposition.

Definition. We shall say that a finite cyclic group $A$ is weakly determined by its automorphism group if for any finite cyclic group $B \varsubsetneqq A$ the condition Aut $A \cong$ Aut $B$ implies that the number of nonzero $B_{p}$ is greater than the number of nonzero $A_{p}$.

Let us note that if for a group $A \in \mathcal{Q D}_{1}$ and $p \in \mathbb{P}$ we have $A_{p}=0$ then group $\mathrm{t}(A)$ is $p$-divisible, and so the groups $A$ and $A / \mathrm{t}(A)$ are simultaneously $p$-divisible or not $p$-divisible.

Theorem 3. A group $A \in \mathcal{Q D}_{1}$ is determined by its automorphism group in the class $\mathcal{Q D}_{1}$ if and only if $\mathrm{t}(A)$ is a cyclic group (possibly equal to zero) which is weakly determined by its automorphism group and $p A \neq A$ for all $p \in \mathbb{P}$ such that $A_{p}=0$.

Proof. A quotient divisible group of rank 1 with non-zero $p$-components is not determined by its automorphism group in the class $\mathcal{Q} \mathcal{D}_{1}$ because automorphism groups of all such quotient divisible groups are isomorphic to each other as it follows from Proposition 2.

Let us suppose that group $A \in \mathcal{Q D}_{1}$ has infinitely many nonzero $p$-components and its cocharacteristic $\chi=\left(m_{p}\right)_{p \in \mathbb{P}}$ contains at least one symbol $\infty$. Let us define $\varphi=\left(h_{p}\right)_{p \in \mathbb{P}} \neq \chi$ by setting

$$
h_{p}=\left\{\begin{array}{cl}
m_{p} & \text { if } m_{p} \neq \infty \\
0 & \text { if } m_{p}=\infty
\end{array}\right.
$$


Then for any group $B \in \mathcal{Q D}_{1}$ with cocharacteristic $\varphi$ we have $B / \mathrm{t}(B) \cong \mathbb{Q}$ and $B_{p} \cong A_{p}$ for every $p \in \mathbb{P}$. Taking into account Proposition 1 , it follows from the isomorphisms

$$
\operatorname{Aut}(A / \mathrm{t}(A)) \cong Z(2) \times \prod_{\aleph_{0}} Z \cong \operatorname{Aut} \mathbb{Q} \cong \operatorname{Aut}(B / \mathrm{t}(B))
$$

that Aut $A \cong$ Aut $B$. Then group $A$ is not determined by its automorphism group in $\mathcal{Q D}_{1}$.

Let us assume that group $B \in \mathcal{Q D}_{1}$ has infinitely many nonzero $p$-components and its cocharacteristic $\varphi$ does not contain symbols $\infty$. Taking into account the case considered in the beginning of the proof, we may assume that $\varphi$ contains at least one symbol 0 . Then $\varphi$ can be obtained from some characteristic $\chi \neq \varphi$ using the procedure described in the previous paragraph. Therefore for every group $A \in \mathcal{Q D}_{1}$ with cocharacteristic $\chi$ we have $A \supsetneqq B$ and Aut $A \cong$ Aut $B$. Hence $B$ is not determined by its automorphism group in the class $\mathcal{Q D}_{1}$.

It remains to consider the case when the group $A \in \mathcal{Q D}_{1}$ has a finite number of nonzero $p$-components, i.e., when the group $\mathrm{t}(A)$ is cyclic (then $A$ splits). It follows from Proposition 1 and from the uniqueness (up to isomorphism) of the decomposition of a group into the restricted direct product of indecomposable cyclic groups that isomorphism Aut $A \cong$ Aut $B$ with $B \in \mathcal{Q D}_{1}$ is possible only in the case when $\mathrm{t}(B)$ is a cyclic group, Aut $(A / \mathrm{t}(A)) \cong \operatorname{Aut}(B / \mathrm{t}(B))$ and $\operatorname{Aut}(\mathrm{t}(A)) \cong \operatorname{Aut}(\mathrm{t}(B))$. We denote the set of all $p \in \mathbb{P}$ for which $A_{p} \neq 0$ by $L$ and the set of all $p \in \mathbb{P}$ for which the group $A / \mathrm{t}(A)$ is $p$-divisible by $X$ (then $L \subset X$ ).

1) Suppose that equality $A_{p}=0$ does not imply $p A \neq A$, i.e., that set $X$ strictly contains $L$. Let us choose a set $Y \subset \mathbb{P}$ so that $L \subset Y, Y \neq X$ and $|Y|=|X|$. If $R$ is the subring of the field $\mathbb{Q}$ generated by elements $\frac{1}{q}$ such that $q \in Y$ then for the group $B=R \oplus \mathrm{t}(A) \in \mathcal{Q D}_{1}$ we have $A \nsubseteq B$ (because $A / \mathrm{t}(A) \nsubseteq R$ ) and

$$
\operatorname{Aut}(B / \mathrm{t}(B)) \cong \operatorname{Aut} R \cong Z(2) \times \prod_{|X|} Z \cong \operatorname{Aut}(A / \mathrm{t}(A)),
$$

whence we obtain Aut $B \cong$ Aut $A$. This means that the group $A$ is not determined by its automorphism group in the class $\mathcal{Q D}_{1}$.

2) Now assume that $X=L$ but the group $\mathrm{t}(A)$ is not weakly determined by its automorphism group. Then there is a finite cyclic group $G ¥ \mathrm{t}(A)$ such that Aut $G \cong \operatorname{Aut}(\mathrm{t}(A))$, and the number of non-zero primary components of $G$ does not exceed $|L|$. Let us choose a set $Y \subset \mathbb{P}$ so that it contains all $q \in \mathbb{P}$ for which $G_{q} \neq 0$ and so that $|Y|=|L|$. If $R$ is the subring of the field $\mathbb{Q}$ generated by elements $\frac{1}{q}$ with $q \in Y$ then for the group $B=R \oplus G \in \mathcal{Q D}_{1}$ we have $B \nsucceq A$ and $\operatorname{Aut}(B / \mathrm{t}(B)) \cong Z(2) \times Z^{|L|} \cong \operatorname{Aut}(A / \mathrm{t}(A))$, whence we obtain Aut $B \cong$ Aut $A$. Consequently, in this case the group $A$ is again not determined by its automorphism group in $\mathcal{Q D} \mathcal{D}_{1}$.

3) Finally, suppose that $X=L$ and the group $\mathrm{t}(A)$ is weakly determined by its automorphism group. Let Aut $A \cong$ Aut $B$ with $B \in \mathcal{Q D}_{1}$. Then it follows from the isomorphism $\operatorname{Aut}(A / \mathrm{t}(A)) \cong$ $\operatorname{Aut}(B / \mathrm{t}(B))$ that the set of all $q \in \mathbb{P}$ such that $B / \mathrm{t}(B)$ is a $q$-divisible group has cardinality $|L|$. This means that $B$ has at most $|L|$ non-zero primary components. Then $\operatorname{Aut}(\mathrm{t}(A)) \cong \operatorname{Aut}(\mathrm{t}(B))$ implies the isomorphism $\mathrm{t}(A) \cong \mathrm{t}(B)$. Thus for every $p \in L$ we have $B_{p} \neq 0$, and hence $B / \mathrm{t}(B)$ is a $p$-divisible group. Therefore $A / \mathrm{t}(A) \cong B / \mathrm{t}(B)$ and so $A \cong B$. We obtain that group $A$ is determined by its automorphism group in the class $\mathcal{Q D}_{1}$. The theorem is proved.

The theorem just proved implies that a quotient divisible group of rank 1 with cocharacteristic

$$
\chi=\left(m_{2}, m_{3}, m_{5}, \ldots\right)
$$


is determined by its automorphism group in the class $\mathcal{Q} \mathcal{D}_{1}$ if and only if $\chi$ does not contain symbols 0 and $m_{p}=\infty$ for almost all $p \in \mathbb{P}$. The finite $m_{p}$ defines a cyclic group which is weakly determined by its automorphism group.

It can be verified directly that if $n \leqslant 50$ then $Z(n)$ is weakly determined by its automorphism group for $n=1,5,8,11,13,16,17,23,24,25,29,31,32,37,41,47$. From this we immediately obtain examples of quotient divisible groups of rank 1 which are determined by their automorphism groups in the class $\mathcal{Q D}_{1}$. For instance, at $n=5$ and $n=24$ we have quotient divisible groups of rank 1 with cocharacteristics $(\infty, \infty, 1, \infty, \ldots, \infty, \ldots)$ and $(3,1, \infty, \infty, \ldots, \infty, \ldots)$, respectively.

The work of the second author was supported by the Ministry of Science and Higher Education of Russia (assignment no. 1.13557.2019/13.1).

\section{References}

[1] H.Leptin, Abelsche p-gruppen und ihre Automorphismengruppen, Math. Z., 73(1960), $235-253$.

[2] W.Liebert, Isomorphic automorphism groups of primary Abelian groups, In: Abelian Group Theory (Proceedings of the 1985 Oberwolfach Conference), Gordon and Breach, New York, 1987, 9-31.

[3] V.K.Vildanov, Determinability of a completely decomposable torsion-free Abelian group of rank 2 by its automorphism group, Vestn. Nizhegorod. Univ. N. I. Lobachevskogo, (2011), no. 3(1), 174-177 (in Russian).

[4] V.K.Vildanov, Determinability of a completely decomposable block-rigid torsion-free Abelian group by its automorphism group, J. Math. Sci., 197(2014), no. 5, 590-594.

[5] L.Fuchs, Infinite Abelian groups, Vols. 1 and 2, Academic Press, New York, 1970, 1973.

[6] O.I.Davydova, Rank-1 quotient divisible groups, J. Math. Sci., 154(2008), no. 3, 295-300.

[7] A.V.Tsarev, T-rings and quotient divisible groups of rank 1, Vestn. Tomsk. Gos. Univ., Mat. Mekh., 4(24)(2013), 50-53 (in Russian).

[8] K.Ireland, M.Rosen, A classical introduction to modern number theory, Springer, New York, 1990. 


\section{Об определяемости факторно делимой абелевой группы ранга 1 своей группой автоморфизмов}

Вадим К. Вильданов

Нижегородский государственный университет им. Лобачевского

Гагарина, 23, Нижний Новгород, 603950

Россия

Егор А. Тимошенко

Томский государственный университет

Ленина, 36, Томск, 634050

Россия

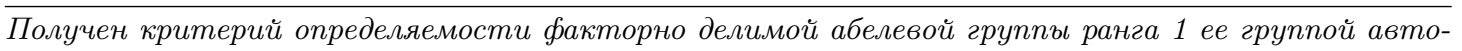
морфизмов в классе факторно делимых абелевых групп ранга 1.

Ключевые слова: определяемость, факторно делимая группа, группа автоморфизмов. 